\title{
High-resolution optical coherence tomography with fiber induced broadband source and process algorithm for oral cancer study
}

\author{
I-Jen Hsu ${ }^{a}$, Chih-Wei Lu ${ }^{a}$, Chih-Chung Yang ${ }^{a}$, Jun-Ping Chiang ${ }^{\mathrm{b}}$, Chii-Wann Lin ${ }^{\mathrm{c}}$ \\ ${ }^{a}$ Department of Electrical Engineering and Graduate Institute of Electro-Optical Engineering, National \\ Taiwan University, 1, Roosevelt Road, Sec. 4, Taipei, Taiwan, R.O.C. \\ (phone) 886-2-23657624（fax) 886-2-23652637（e-mail) ccy@cc.ee.ntu.edu.tw \\ ${ }^{\mathbf{b}}$ Department of Dentistry, National Taiwan University, Taipei, Taiwan, R.O.C. \\ ${ }^{\mathrm{c}}$ Graduate Institute of Bioengineering, National Taiwan University, Taipei, Taiwan, R.O.C.
}

\begin{abstract}
A polarization controllable optical coherence tomography (OCT) system was built with the broadband source generated with femtosecond Ti:sapphire laser pulses in an ordinary fiber and a photonic crystal fiber. Spectral broadening in such fiber originated from self-phase modulation, four-wave mixing, Raman scattering, and other nonlinear-optics effects. Two different mode-locked Ti:sapphire lasers with $100 \mathrm{fsec}$ and $12 \mathrm{fsec}$ pulses were used. The generated spectral shape and width were compared in terms of the application to the OCT system. The relationship between the OCT resolution and the source spectrum shape was studied. Also, an algorithm was built for increasing the effective longitudinal resolution in data processing. The scheme of this algorithm meant to separate the contribution of the central portion from those of the tails in the interference fringe envelope. By removing the tail contribution to the scanning results, the effective longitudinal resolution was improved. Such a procedure is particularly important when the light source spectrum is not a well-defined shape. This procedure involved in the computation of a matrix inversion. The OCT system and the process algorithm were used for oral cancer study. Features of oral cancer were well identified. A probe was also fabricated for in vivo scan of oral tissues.
\end{abstract}

Keywords: optical coherence tomography, nonlinear effects in fiber, oral cancer, resolution

\section{INTRODUCTION}

Optical coherence tomography (OCT) and optical Doppler tomography (ODT) have been widely studied for high-resolution subsurface tissue scanning ${ }^{1,2}$. They were developed for diagnosing various tissue abnormalities either on skin or inside-organ through endoscopes. OCT or ODT uses the technique of low-coherence interferometry. Typically, Michelson interferometers were built for implementing OCT scanning. Because the longitudinal resolution in OCT scanning, which is equal to the width of interference fringe envelope, is inversely proportional to the light source bandwidth, pursuing means for generating stable broadband light source, either coherence or incoherent, is crucially important for OCT development. In the past, most OCT systems utilized super-luminescence light-emitting diodes as sources. Such a light source, either centered around 850 or $1300 \mathrm{~nm}$, typically has a spectral width about $30 \mathrm{~nm}$ and pig-tailed output power less than $1 \mathrm{~mW}$. This spectral width could lead to only around 10 or $25 \mu \mathrm{m}$ in depth resolution in free space. Besides, the low available power resulted in relatively low sensitivity. The combination of two super-luminescence diodes of different central wavelengths was also used for broadening the effective spectral width and hence higher resolution. Recently, based on a broadband semiconductor source (an AFC product) of $1310 \mathrm{~nm}$ in central wavelength, $80 \mathrm{~nm}$ in spectral width, and several $\mathrm{mW}$ in power, a depth resolution around $10 \mu \mathrm{m}$ has been reported. Portable semiconductor broadband sources with even larger spectral widths and higher output powers deserve further developments. On the other hand, with ultrashort Ti:sapphire laser pulses, the corresponding $260 \mathrm{~nm}$ spectral width resulted in a depth resolution as small as $1.5 \mu \mathrm{m}$. Also, high laser output can always provide sensitivity higher than $120 \mathrm{~dB}$. To achieve deeper penetration, a mode-locked Cr:forsterite laser with central wavelength at $1280 \mathrm{~nm}$, was used to achieve $5.7 \mu \mathrm{m}$ longitudinal resolution in free space. In this work, the 50-nm spectral width from the laser was broadened to around $150 \mathrm{~nm}$ after pulse propagation in a dispersion-shifted fiber. The spectral broadening was attributed to self-phase modulation, which inter-played with normal group-velocity dispersion.

In our OCT experiments, we used spectral broadening of femtosecond Ti:sapphire lasers, based on nonlinear optics in a single-mode fiber and a photonic crystal fiber, which are Kerr media ${ }^{3}$, for broadband source generation. Widely observed nonlinear effects in the normal group-velocity dispersion range of a single-mode fiber include self-phase modulation, Raman scattering, Brillouin scattering, and four-wave mixing. With the Kerr effect in fiber, the refractive index increases linearly with optical intensity such that nonlinear phase modulation or chirp is generated for a pulsed signal. Through this self-phase modulation process, the spectrum of a short pulse is broadened. Besides self-phase modulation, stimulated Raman scattering and four-wave mixing in fiber can broaden spectrum. Particularly, stimulated Raman scattering typically results in red shift 
of signal spectrum and leads to spectral asymmetry. Recently, by tapering fiber or using a photonic crystal fiber, extremely broadband generation with output wavelength ranging from visible to near infrared has been reported. Because mode-locked Ti:sapphire lasers with output pulse widths in the sub-100 fsec range are quite popular, this approach should be quite useful for many laboratories. We used two different mode-locked Ti:sapphire lasers with $100 \mathrm{fsec}$ and $12 \mathrm{fsec}$ pulses in our experiments. The generated spectral shape and width were compared in terms of the application to the OCT system. The relationship between the OCT resolution and the source spectrum shape was studied. Also, an algorithm was built for increasing the effective longitudinal resolution in data processing. By removing the tail contribution to the scanning results, the effective longitudinal resolution was improved. Such a procedure is particularly important when the light source spectrum is not a well-defined shape. The built OCT system and the process algorithm were used for oral cancer study. Features of oral cancer were well identified.

\section{EXPERIMENTAL PROCEDURES}

Two different mode-locked Ti:sapphire lasers with $100 \mathrm{fsec}$ and $12 \mathrm{fsec}$ pulses were used in our experiments. The lasers were pumped with the second-harmonics of diode-pumped $\mathrm{Nd}: \mathrm{YVO}_{4}$ lasers. The pulses were end-coupled into a single-mode fiber (3M, FS-SN-4224) and a photonic crystal fiber in different experiments. The pulsed signal from the fiber output end was directed to a free-space OCT system. The output spectra of the single-mode fiber were asymmetrically broadened. The asymmetric output spectra may result from several possible mechanisms, including asymmetric input pulses, significant stimulated Raman scattering, and four-wave mixing. Because the central wavelengths have been red-shifted, it is supposed that stimulated Raman scattering plays an important role here. The output spectrum of the photonic crystal fiber was extremely broadened with wavelength ranging from visible to near infrared. After coupling the laser pulses into a singlemode fiber, the spectra of the two different lasers can be broadened to about 50 and a few hundred nm, respectively. The spectral width and shape were controllable by changing the input power of the fiber, which results in different longitudinal resolution of the OCT system. The generated spectral shape and width were compared in terms of the application to the OCT system. The relationship between the OCT resolution and the source spectrum shape was studied. In the OCT system, a lens with $3 \mathrm{~mm}$ in focal length was used for focusing light beam onto samples under in vitro scan. The beam size at the focal point was estimated to be around $5 \mu \mathrm{m}$, which corresponded to the lateral resolution. The high lateral resolution was chosen at the expense of a short depth of focus or depth range of scanning, which was estimated to be just a few hundred $\mu \mathrm{m}$. The available power for sample illumination could be as high as $100 \mathrm{~mW}$. In polarization-dependent tomography operation, circularly polarized signals were applied onto samples. A polarizer was then used before the photo-detector for polarizationgated scan. The sensitivity was estimated to be higher than $100 \mathrm{~dB}$.

\section{DATA PROCESSING}

Because the spectrum of the light source generated through nonlinear optics effects in fiber is not a well-defined shape, there usually exists some effects of side lobes and tails in the interference fringe envelope that may cause artifacts and reduce the resolution of the OCT system. We have studied the relationship between the OCT resolution and the source spectrum shape. Also, an algorithm was built for eliminating the effects of side lobes and increasing the effective longitudinal resolution in data processing. The scheme of this algorithm meant to separate the contribution of the central portion from those of the tails in the interference fringe envelope. By removing the tail contribution to the scanning results, the effective longitudinal resolution was improved. The idea of this algorithm is to consider the signal at each point of an OCT image as a combined contribution of the light scattered from the observed position and its neighborhood. It is actually a transformation via a function $f(z)$,

$$
I\left(z_{0}\right)=\int_{z_{i}}^{z_{f}} \tilde{I}(z) f\left(z-z_{0}\right) d z,
$$

where $I(z)$ denotes the detected signal at position $z, \tilde{I}(z)$ denotes the intensity of light scattered from point $z$, and $f(z)$ is a function that describes the interference fringe envelope and is normalized to have value of unity at zero point,

$$
f(0)=1 \text {. }
$$

Because an OCT image was composed by discrete pixels, Eq.(1) can be replaced by

$$
I_{i}=\sum_{j} F_{i j} \tilde{I}_{j} \Delta z,
$$

where $i$ and $j$ indicate the pixel number and $F$ is a matrix that represents the transformation between original and processed data. The procedure can be accomplished by finding an inverse transformation of the original image.

$$
\tilde{I}_{i}=\sum_{j} F_{i j}^{-1} I_{j} / \Delta z .
$$

In principle, such a process can improve the resolution of an OCT system beyond the limitation of the bandwidth of its light source. Fig. 1 shows the OCT images of a piece of human oral mucosa, in which cancerous cells were identified. After 
processing through our algorithm, the detailed features in the resulted image (Fig. 2(b)) were clearer than the original image (Fig. 2(a)).

\section{CONCLUTIONS}

In summary, we have built a polarization dependent OCT system with its broadband sources generated with nonlinear optics process in a single-mode fiber and a photonic crystal fiber. We used two different mode-locked Ti:sapphire lasers with 100 fsec and $12 \mathrm{fsec}$ pulses in our experiments. By controlling the input power, we have studied the relationship between the OCT resolution and the source spectrum shape. To eliminate the effects of side lobes and tails in the interference fringe envelope and improve the resolution, we proposed an algorithm for data processing. The built OCT system and the process algorithm were used for oral cancer study. Features of oral cancer cells were well identified. The detailed features become clearer after the process through our algorithm.

\section{ACKNOWLEDGMENTS}

This research was supported by National Health Research Institute, The Republic of China, under the grants of NHRI-GTEX89E819L.

\section{REFERENCES}

1. D. Huang, E. A. Swanson, C. P. Lin, J. S. Schuman, W. G. Stinson, W. Chang, M. R. Hee, T. Flotte, K. Gregory, C. A. Puliafito, and J. G. Fugimoto, "Optical coherence tomography," Science 254, pp. 1178-1181, 1991.

2. S. Yazdanfar, A. M. Rollins, and J. A. Izatt, "Imaging and velocimetry of the human retina circulation with color Doppler optical coherence tomography," Opt. Lett. 25, pp. 1448-1450, 2000.

3. G. P. Agrawal, Nonlinear Fiber Optics, 2nd ed. Academic, San Diego, California, 1995.

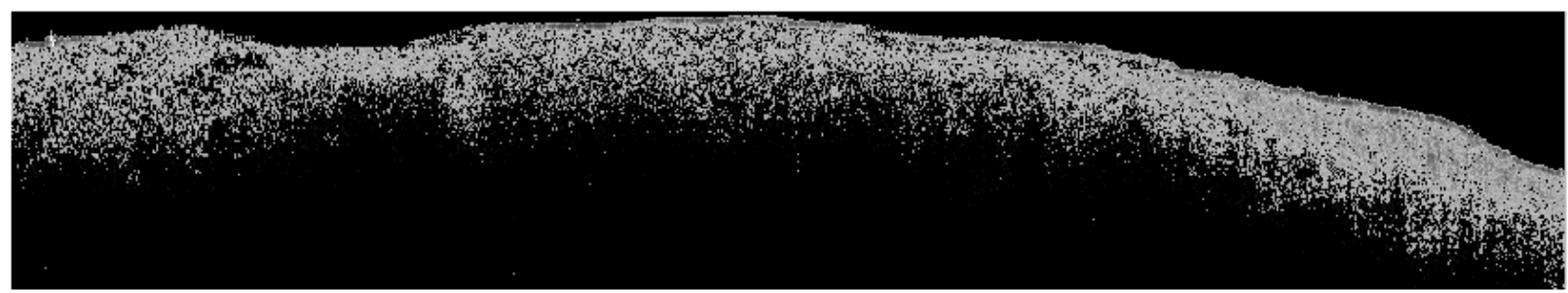

Fig. 1: An OCT scan picture of a piece of human oral mucosa sample with cancer cells.

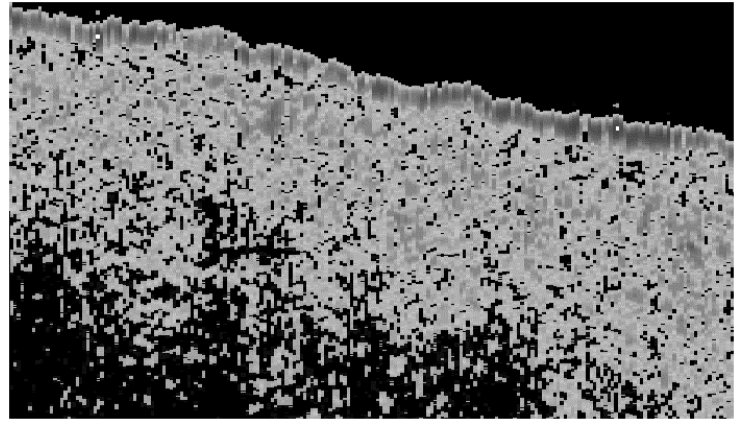

(a)

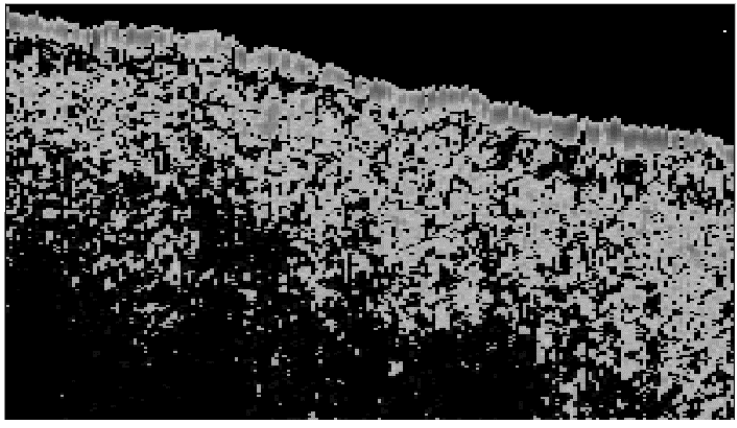

(b)

Fig. 2: A part of the OCT image of the cancerous human oral mucosa (a) becomes clearer in detailed features after the process through our algorithm (b). 